\begin{tabular}{|l|l|l|}
\hline \multirow{4}{*}{$\begin{array}{l}\text { INTERNATIONAL } \\
\text { ENGINEERING, } \\
\text { SCIENCE AND } \\
\text { EDUCATION } \\
\text { INESEG }\end{array}$} & $\begin{array}{l}\text { Middle East Journal of Science } \\
\text { GROUP }\end{array}$ & $\begin{array}{l}\text { Published online August 28, 2017 } \\
\text { doi: } 10.23884 / \text { mejs.2017.3.1.04 } \\
\text { ISSN: } 2536-5312\end{array}$ \\
\hline
\end{tabular}

\title{
THE EFFECT OF CHAOS THEORY ON THE PREDICTABILITY OF THE ATMOSPHERE
}

\author{
Fatma Aydogmus ${ }^{* 1}$, Eren Tosyali ${ }^{2}$ \\ ${ }^{* 1}$ Istanbul University, Department of Physics, Istanbul, Turkey \\ ${ }^{2}$ Istanbul Bilgi University, School of Advanced Vocational Studies, Istanbul, Turkey \\ *fatmaa@istanbul.edu.tr
}

\begin{abstract}
It is known that atmosphere is a dynamical system that constantly changing and moving. It is a very difficult problem to completely determine and explain the change in the atmosphere. But the mathematical models can help us to understand and explain the dynamical processes in the atmosphere. Examination of the movements of the atmosphere is one of the most exciting applications of chaos theory. The main goals of this presentation will be try to discuss the effect of chaos theory on the predictability of the atmosphere. For this purpose, we focus on one of the most specific examples of atmosphere events, is global climate change.
\end{abstract}

Key words: atmosphere, chaos, autonomous

\section{Introduction}

It is well known that a periodic motion is predictable and repeats itself. It returns to a specific position with the same velocity. But a regular motion defines only a small part of all possible sustained motion. Most systems exhibit irregular behaviors. The irregular behaviors are produced by a deterministic system and unpredictable in the long-range. Chaos is about the irregular behaviors of a deterministic system. Contrary to popular belief, chaos theory interest deterministic systems, i.e. the initial condition is known exactly and the future state can be predicted theoretically [1]. But these systems are predictable just for a while and then become to random. Today it is known that chaos theory play an important role in nonlinear science. It has applications in several disciplines, including physics, mathematics, sociology, environmental science, meteorology.

Chaotic behaviors also exist in many natural systems, such as atmosphere. It is known that atmosphere is an autonomous dynamical system [2]. Because of the existence many variables, such systems are rather difficult to model and study numerically. The numerical methods in solving such problems have become more relevant with the increase of computational possibilities. The development 
of computer technology together with theoretical observations makes it possible to study the autonomous dynamic structure of the atmosphere.

The signs of the global climate change are followed with anxiety. Scientist try to best to improve some mathematical tools and simulation model to predict not only global but also local effects of the global climate change. As well known, the near future of the atmospheric system is determined by physical laws and predictable but it is difficult to extend of this predictability of the daily forecasting up to two or three weeks [3]. The long-term forecasting expands predicting the average values in a definite period, such as the monthly averaged anomalies of temperature [4]. The annual temperature anomalies between 1880-2014 are investigated in the paper exemplary. The used data are taken from the CDIAC.

\section{Lorenz Model}

As well known, it is a very difficult problem to completely determine the dynamical structure of the atmosphere. But the mathematical models can help us to understand and explain the dynamical processes in the atmosphere. Such a model was created by Lorenz in 1961. The Lorenz equations define an autonomous dynamical system [5].

Lorenz modeled the atmospheric circulation as a 2D flow of an incompressible fluid in a 3D heated layer. Starting from the hydrodynamic equations, he arrived at a set of three nonlinear autonomous differential equations;

Lorenz was aware that the atmosphere behaved as a turbulent fluid and governed by a nonlinear differential equations system that highly sensitive to initial conditions. He has reduced the complex equations system to a simple model with only three variables, $x, y, z$. These three variables, and represent the state of the Earth's atmosphere, the state space is R3 [6].

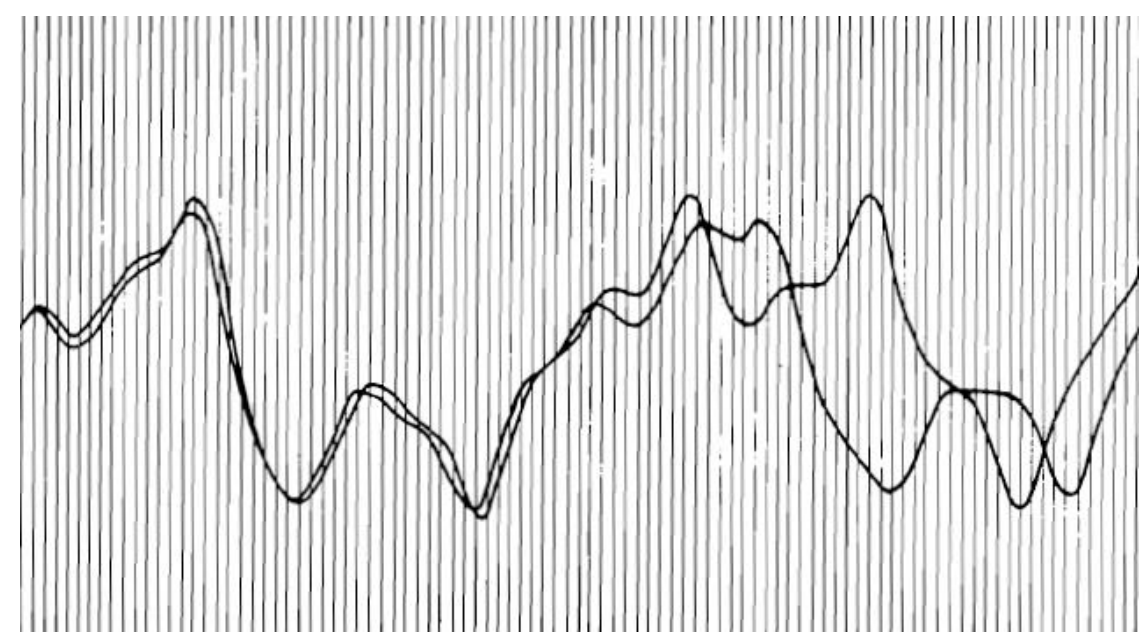

Figure 1. From Lorenz's 1961 printouts. 
Two random points near to each other represent almost similar weather conditions. From nearly the same starting point, Lorenz saw his computer produce patterns that grew farther and farther away until all similarity disappeared.

\section{Global Climate Change}

It is well known that the global climate change is one of the most important interests of the scientist. According to the geological and stratigraphic researches, there have been important global climate changes in our world as far as the present day[7]. For example, between 11,000 and 10,000 years $(9,000-$ $8,000 \mathrm{BC}$ ) has been a rapid climate change[8]. Recent studies have shown that a change of 6 degrees centigrade in the northern hemisphere temperature causes severe changes in the climate. Increase of sea water levels, annual temperature increase, reduction of glaciers and snow cover on high mountain peaks as glaciers melt away towards the poles, propagation of tropical plants and fish to the poles, decrease of delicate bird species sensitive to airborne contaminants and so on are some important signs and effects of global climate change [9]. Of course, one of the most important causes is human activity. Scientist try the best to improve some mathematical tools and simulation models to predict not only global but also local effects of the global climate change. In the paper, we deal annual temperature anomalies as one of the effects of global climate change.

\section{Numerical Results}

Below, the plots of annual and hemispheric annual temperature anomalies from land and ocean observations are given between 1880-2014. The used data are taken from the CDIAC [10]. The plots show us that the temperature anomalies are increasing year by year.

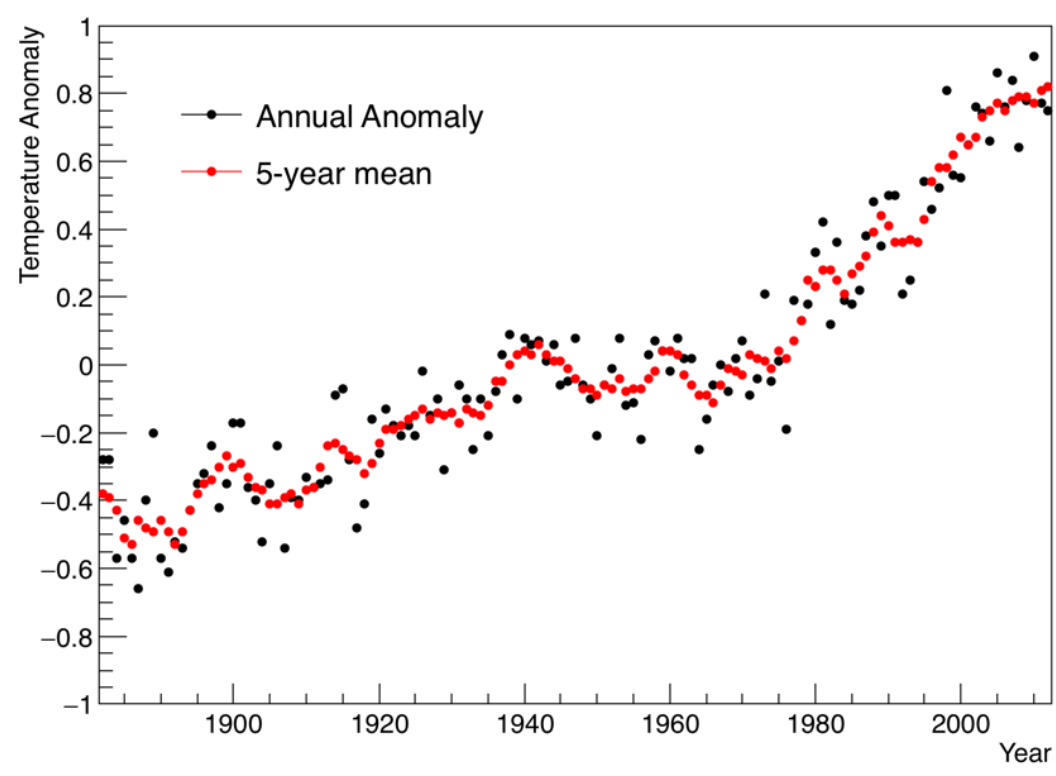

Figure 2. Annual Temperature Anomalies from Land Observations, 1880-2014 


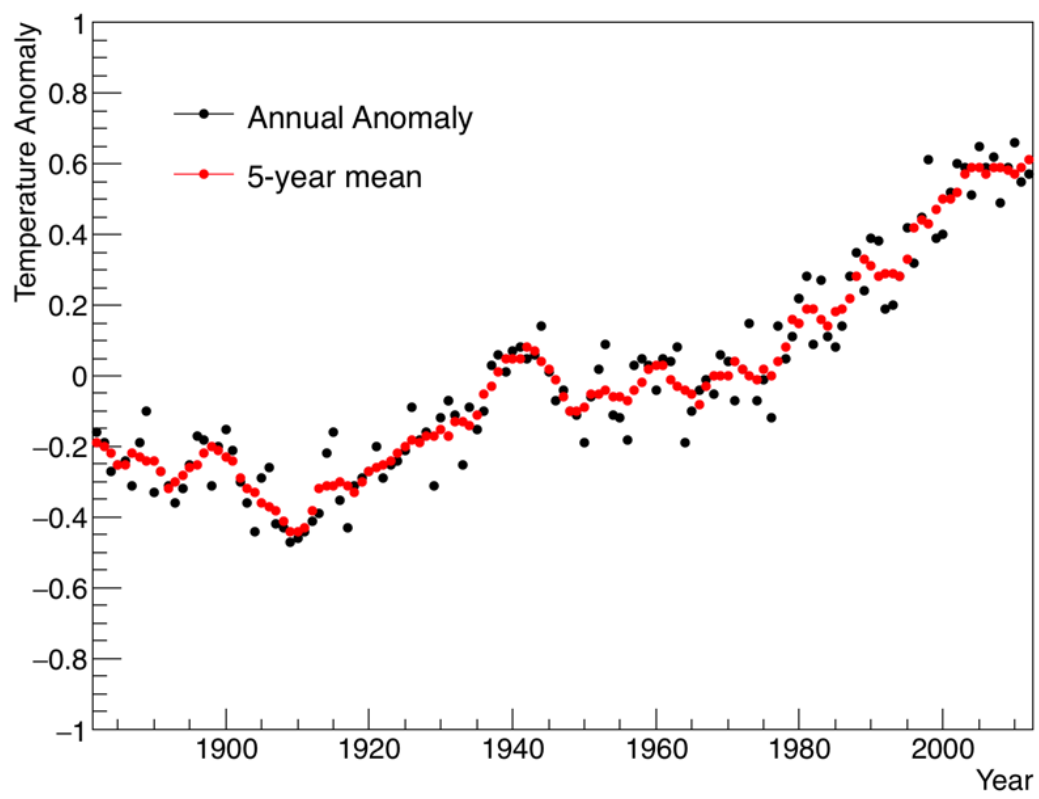

Figure 3. Annual Temperature Anomalies from Land and Ocean Observations, 1880-2014

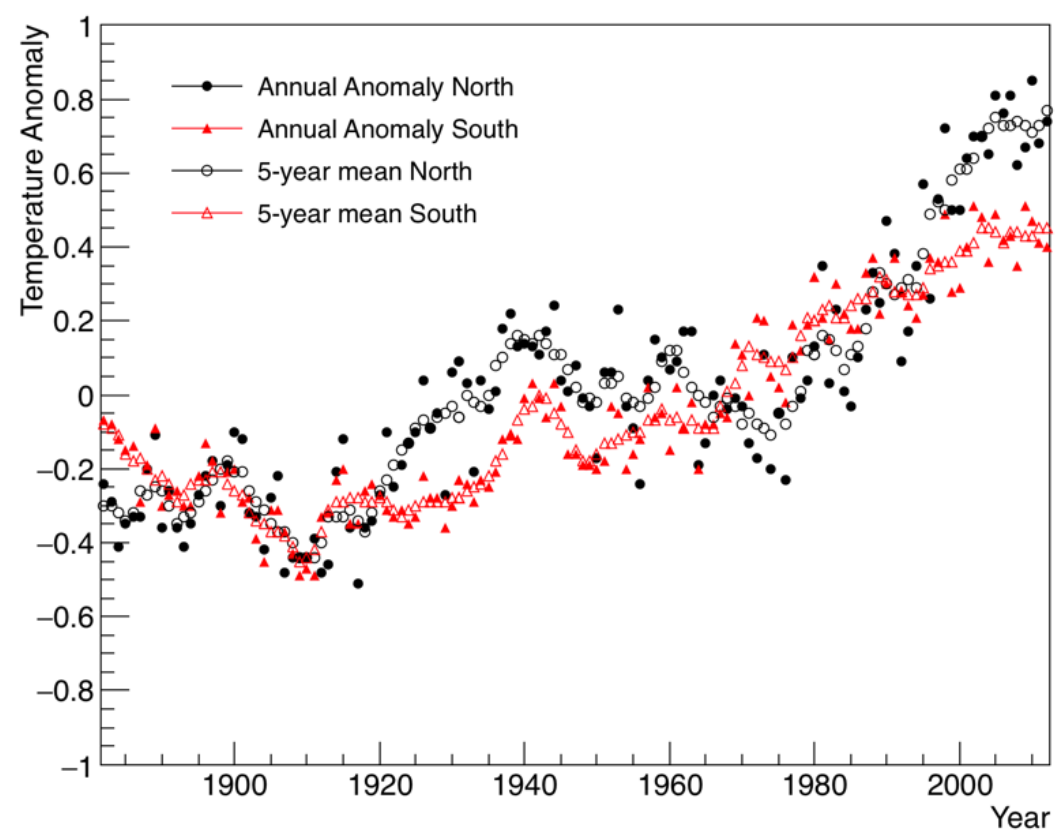

Figure 4. Hemispheric Annual Temperature Anomalies from Land and Ocean Observations, 1880-2014 


\section{Conclusion}

The evolution of the movements in the atmosphere may be entirely different for the two initial values with small differences. In other words, the very small differences in the weather conditions can cause very different weather conditions that can not be predicted in the atmosphere. The limit of predictability of the small differences in the initial conditions of the atmosphere is also called the "butterfly effect". The biggest problem is that the meteorological parameters can not be accurately measured at all. Temperature, pressure and similar variables are measured approximately at very infrequent locations with the meteorological instruments. These missing and incorrectly measured parameters are used by meteorologists as initial values in weather forecasts. In this case, there can be a difference between the actual values and the values used as initial values. In such a case, even if all the physical laws that controlling the atmosphere are known, the prediction may be entirely different due to the chaotic attractor. This is a feature of chaotic systems; the difference between the actual and the used initial values grows over time in the models and limits our ability to predict the system's movements [2].

In this paper, we consider global climate change as one of the most specific examples of atmosphere events. In the light of the plots, we can conclude that the temperature anomalies are increasing year by year. These anomalies can affect the dynamical structure of atmosphere. Such effects may cause the several times larger chaotic fluctuations in the nature of atmosphere[1]. This might allow one to establish some theories to understand the effects of the global climate change on certain sensitive structures. As is known, nonlinear dynamical systems are governed by rather complex physical processes. The atmosphere is a highly dynamical system. Due to the nonlinear structure of atmosphere, the climate change should sensitively change the global climate behaviours from the regularity to chaos. Ongoing studies will bring a better description on this subject.

\section{References}

[1] Aydogmus F., Akdeniz G., Global Climate Change And Human With A Syborg Science Doctrin, IV. International Symposium on Sciences of Atmosphere, Istanbul Teknik University, (2008), pp.437-443.

[2] Aydogmus F., Tosyali E., (2015), Global Climate Change in Consideration of Chaos Theory, 9th International Physics Conference of the Balkan Physical Union - BPU9, İSTANBUL, TÜRKIYE, 24-27 Ağustos 2015, .502.

[3] Jifan C., (1989), Predictability of the Atmosphere, Advances in Atmospheric Sciences, Vol.6, No.3.

[4] Kaper H. and Engler H., (2013), Mathematics and Climate, ISBN: 978-1-61197-260-3.

[5] Lorenz E., (1963), "Deterministic nonperiodic flow". Journal of the Atmospheric Sciences. 20 (2): $130-141$.

[6] Palmer,T.N.,(1998 ), Climate change from a nonlinear dynamical perspective., J.Clim.,12,575591. 
Middle East J. of Science (2017) 3(1):26-31

[7] Palmer,T.N.,(2000), Uncertainty in Forecast of Weather and Climate, Rep.Prog.Phys.,63,71-116.

[8] Tel T. and Gruiz M. (2006), Chaotic Dynamics, Cmabridge University Press.

[9] Retrieved from www.climate.nasa.gov.

[10] Retrieved from www.cdiac.gov. 